\title{
Tangence
}

\section{Lire la Bible, une aventure jamais terminée}

\section{Jérôme Longtin}

Numéro 35, mars 1992

Des écritures à lire

URI : https://id.erudit.org/iderudit/025699ar

DOI : https://doi.org/10.7202/025699ar

Aller au sommaire du numéro

Éditeur(s)

Tangence

ISSN

0226-9554 (imprimé)

1710-0305 (numérique)

Découvrir la revue

Citer cet article

Longtin, J. (1992). Lire la Bible, une aventure jamais terminée. Tangence, (35), 59-73. https://doi.org/10.7202/025699ar d'utilisation que vous pouvez consulter en ligne.

https://apropos.erudit.org/fr/usagers/politique-dutilisation/ 


\title{
Lire la Bible, une aventure jamais terminée
}

\author{
Jérôme Longtin
}

Les Anciens écrivaient pour être lus! Cela peut sembler un lieu commun mais il est utile de le rappeler. On ne trouve dans la Bible ni Journal intime, ni Mémoires, ni notes personnelles. Même un écrit aussi original que Qobélet suppose un destinataire auquel l'auteur s'adresse parfois à la deuxième personne ${ }^{1}$.

Dès lors se pose le problème de la lecture, c'est-à-dire de la réception du texte par tout lecteur potentiel. Comment les destinataires originaux comprenaient-ils le texte qui leur était adressé? Comment, par la suite, d'autres lecteurs auxquels l'auteur n'avait pas songé en écrivant reçoivent-ils son texte? Comment peut être franchie la distance entre le texte et un lecteur qui lui est en quelque sorte étranger?

L'acte de lire suppose nécessairement le franchissement d'une distance entre le texte, comme objet, et le lecteur comme sujet. La distance a parcourir peut être plus ou moins grande. Le premier lecteur - celui à qui le texte est adressé directement - aborde le texte dans une position d'immédiateté qui ne se reproduira jamais plus. Ce qui ne veut pas dire qu'il ait automatiquement du texte et de toutes ses potentialités une compréhension supérieure ou supposée plus authentique, car ce premier lecteur aborde aussi le texte avec ses présupposés et ses questions. Son avantage par rapport aux lecteurs subséquents - si avantage il y a - se situe au niveau de la compréhension plus immédiate du code employé par l'auteur.

Une fois que le destinataire originel a pris connaissance du texte qui lui est adressé, il peut soit l'abandonner comme un objet devenu inutile, soit le conserver. Si on le conserve, c'est qu'on lui reconnaît une valeur permanente, une utilité éventuellement différente de celle de l'origine, mais qui peut se déployer dans le temps. On admet ainsi, au moins implicitement, que le texte n'a pas livré dès la première lecture tout le message dont il était potentiellement porteur. On ouvre la porte à des relectures, ce qui suppose évidemment des relecteurs.

Par exemple: Qo 5,1-7; 6,9-10.14-22; 9,7-10; 11,1-6. 
60

Chacun des relecteurs doit se situer par rapport au texte en tenant compte de la distance qui l'en sépare. Une première attitude possible consiste à nier la distance avec le texte. Le relecteur se situe alors - consciemment ou non - comme s'il était le premier destinataire. Il assume le texte de la même manière que s'il lui était directement adressé. Une deuxième attitude consiste à essayer de reconstituer le milieu originel du texte. Par l'emploi de méthodes appropriées, le relecteur tente de se rappeler le plus possible dans la position historique et culturelle du premier lecteur. Il va à la rencontre du texte. Une troisième attitude consiste à surmonter la distance en transposant le texte dans l'aujourd'hui du relecteur, en adécodant - le langage employé dans le texte pour le réexprimer dans celui du relecteur. Ici, le texte vient à la rencontre de l'actualité du relecteur.

\section{Le phénomène de relecture à l'intérieur de la Bible elle-même}

Il est difficile d'établir avec certitude une chronologie relative des textes bibliques, sinon de manière très générale. Toutes les tentatives donnent lieu à des controverses. Les résultats les plus assurés de la critique sont parfois remis en question une génération plus tard. Le problème de la chronologie des textes rend difficile à cerner le phénomène des * relectures. à l'intérieur des livres bibliques. Pour avancer en terrain sûr, on se contentera ici d'évoquer comment des écrivains bibliques utilisent des textes plus anciens.

Les auteurs des textes consignés dans la Bible n'ont pas exprimé de réflexion théorique sur leur approche des écrits qu'ils lisaient. Si une telle démarche a eu lieu, elle n'a pas laissé de traces qui soient venues jusqu'à nous. Cela ne signifie pas qu'ils ne s'étaient pas posé de questions relatives au sens des textes qui leur parvenaient déjà portés par une tradition.

L'exemple le plus évident de réutilisation de textes antérieurs est celui des deux livres des Chroniques ${ }^{2}$. La critique situe la production de cet ensemble littéraire au début de la période hellénistique, soit vers 300 ap. J.-C. Le peuple juif n'a plus à cette

2 Pour ne pas entrer dans les discussions techniques concernant l'unité ou la pluralité d'auteurs, nous emploierons le terme conventionnel de chroniste pour désigner la ou les personnes responsables de la forme finale et cet ensemble littéraire. 
époque d'autonomie politique; ses membres sont dispersés un peu partout dans les royaumes issus de l'empire d'Alexandre. Même ceux qui sont regroupés autour de Jérusalem, sur le territoire des anciens royaumes de Juda et d'Israël, sont ballottés entre la domination de l'Égypte et celle de la Syrie. Par contre, sur le plan religieux, les juifs jouissent d'une relative autonomie. C'est dans ce domaine que, pour préserver leur identité, il font porter l'essentiel de leurs efforts. À défaut d'institutions politiques, l'accent est mis très fortement sur le temple et son culte, ainsi que sur la Loi perçue comme l'héritage propre d'Israël au milieu des nations.

C'est dans ce contexte que le chroniste entreprend de réécrire l'histoire de son peuple. Pour le faire, il dispose de sources disparues qu'il cite à l'occasion (voir par exemple 1 Ch 29,29; 2 Ch 9,29 ; 12,15; etc.) et surtout de sources qu'il ne cite jamais mais auxquelles nous avons accès: les livres de Samuel et des Rois. À quelques exceptions ${ }^{3}$ près, on peut dire que toute la matière narrative des livres des Chroniques provient d'écrits antérieurs. Et pourtant l'œuvre finale est tout autre chose qu'une pure répétition des textes utilisés; le chroniste n'est pas simplement un compilateur mais un auteur. La manière de procéder du chroniste nous montre, d'une part, qu'il est conscient de la distance qui s'est établie entre les textes dont il dispose et le moment où lui-même les relit et, d'autre part, qu'il croit que ces textes anciens - et les événements qui y sont consignés - ont toujours du sens pour son aujourd'bui. Dans le cas contraire, il aurait plutôt écrit une œuvre entièrement nouvelle, répondant aux attentes et aux besoins de ses lecteurs. Le choix qu'il fait de réutiliser des textes vieux déjà de plusieurs générations atteste qu'à ses yeux les événements passés et les textes qui les racontent sont porteurs d'un sens qui n'est pas épuisé, même si les circonstances historiques originelles ont disparu.

Pour produire l'effet de sens recherché, ces textes anciens ne peuvent cependant plus être réutilisés tels quels. Ils doivent être "actualisés ", c'est-à-dire situés dans le contexte nouveau dans lequel on les relit. En faisant cette opération, le chroniste devient véritablement l'auteur d'une œuvre originale. Son travail consiste à montrer comment les institutions qui assurent la survie et la cohésion de son peuple au moment où il écrit - son aujourd'but - 


\section{2}

ne sont pas des phénomènes passagers; elles s'enracinent au contraire dans l'histoire et font partie de l'héritage national le plus authentique. Pour s'en convaincre, il suffirait de comparer pas à pas la démarche du chroniste avec celle de ses devanciers. Mentionnons, simplement à titre d'exemple, à quel point le chroniste a transformé l'image de David qui apparaissait dans les livres de Samuel et des Rois. De chef militaire et homme politique il devient chef religieux, rassembleur du peuple autour de son Dieu et surtout initiateur du culte dans le temple: à ce sujet le chroniste consacre huit chapitres complets ${ }^{4}$ alors que l'intention de David de construire un sanctuaire est à peine évoquée en $2 \operatorname{Sam} 7,2$.

\section{La traduction comme lieu de relecture et d'actualisation}

Le fait de traduire implique en lui-même une relecture. Rendre dans une langue nouvelle ce que la langue originale exprimait suppose un changement de destinataire: il ne s'agit plus des lecteurs que l'auteur avait directement en vue, mais d'un public nouveau dont on postule qu'il est intéressé à lire le texte traduit à son intention. On présume donc que le document à traduire possède une richesse de sens susceptible de déborder l'intérêt des premiers destinataires pour rejoindre de nouveaux lecteurs appartenant à un autre univers culturel.

Traduire c'est faire franchir au texte une partie de la distance qui le sépare de ses nouveaux destinataires. La traduction tente de combler le fossé qui sépare le texte écrit dans une langue déterminée et le lecteur qui ne la connaît pas. Le traducteur substitue au texte original un texte nouveau dont l'intention explicite est d'exprimer le contenu original dans un langage accessible aux nouveaux destinataires.

La Bible n'échappe pas à cette transformation. De ses origines à nos jours, elle est incontestablement l'œuvre littéraire traduite le plus souvent et dans le plus grand nombre de langues. Il n'est évidemment pas question d'examiner ici l'histoire complète des traductions de la Bible. Nous allons simplement mentionner les premières tentatives pour faire passer la Bible hébraïque dans la langue et la culture du monde grec, ainsi que du monde araméen d'autre part. 
La traduction grecque dite des Septante, réalisée à Alexandrie au cours du troisième siècle avant Jésus-Christ, visait un double but. D'abord fournir à l'importante communauté juive d'Égypte le texte des Écritures hébraïques dans la langue qui était désormais la sienne, lui permettant ainsi de ne pas perdre le contact avec ses origines. Ensuite, mettre la littérature juive à la disposition du public païen intéressé 5 .

Le premier objectif était sans doute le plus important. Les traducteurs ne travaillèrent cependant pas tous selon les mêmes méthodes et les résultats, évalués selon nos critères modernes, sont très inégaux d'un livre à l'autre ${ }^{6}$. L'important pour notre propos est de constater que cette traduction a été reçue par la communauté juive d'Alexandrie - et finalement par l'ensemble des communautés de la Diaspora - comme étant la Parole de Dieu, au même titre que les originaux hébreux et araméens, même si elle ne représentait pas une transcription exacte du texte primitif, mais bien plutôt une adaptation aux besoins nouveaux d'une communauté différente de celle à laquelle les premiers auteurs avaient songé.

Le passage de l'hébreu à l'araméen s'est fait en Palestine et dans les provinces orientales de l'Empire perse puis des royaumes hellénistiques. Le cadre d'origine de ces traductions est d'abord liturgique: c'est dans le culte synagogal qu'est apparu le besoin d'une proclamation des $\vec{E} c r i t u r e s$ intelligible par les personnes présentes ${ }^{7}$. La lecture liturgique étant, par sa nature, proclamation et actualisation, les traducteurs ont intégré au texte lui-même des éléments de commentaires destinés soit à expliciter le texte, soit à édifier les fidèles. Les choix des traducteurs reflètent leurs convictions théologiques et l'évolution de la sensibilité religieuse depuis l'époque de la production du texte original jusqu'à la fixation de la traduction. Ces ouvrages - appelés Targum - ne furent mis par écrit qu'à une époque relativement récente après une longue période de développement oral.

5 Voir par exemple Prologue du Siracide, v. 34-35.

6 Au deuxièmé siècle avant notre ère, l'auteur du Prologue de Ben Sirah juge sévèrement l'ensemble de la traduction telle qu'elle existe de son temps. Il semble cependant croire qu'il n'était pas possible de faire mieux, étant donnée la difficulté qui existe à rendre en grec une pensée exprimée originairement en hébreu ( $c f . v, 19-26)$.

Voir Neh 8,8. 
64

Bien sûr, certaines adaptations faites aux textes originaux surprennent. Elles ne correspondent ni à notre sensibilité, ni à nos habitudes de lecture. Plutôt que d'y voir de pures extravagances ce qui fut longtemps l'opinion des savants occidentaux - il vaut mieux y découvrir le reflet des préoccupations et des interrogations des communautés juives cherchant à rendre actuelle la Parole de Dieu transmise dans la Loi et les Prophètes.

Bien que de manière différente, les traducteurs de la Septante et les Targumistes ont voulu répondre à un même besoin: rendre accessible à leurs contemporains des textes dont la valeur permanente transcendait le contexte immédiat où ils avaient vu le jour. Conscients de la distance séparant ces textes de leur aujourd'hui, l'un et l'autre groupe de traducteurs ont franchi allègrement cette distance afin de rendre aux textes pertinence et actualité pour le public auquel ils s'adressaient.

\section{Le contexte chrétien : le Christ accomplissement des Écritures}

Pour le chrétien, Jésus représente en sa personne l'accomplissement total des Écritures. Tout en reconnaissant dans l'événement Jésus une nouveauté radicale par rapport à tout ce qui avait existé auparavant, les premières générations chrétiennes ont eu à coeur de souligner également la continuité dans la réalisation du plan divin. Aux yeux des croyants, c'est le même Dieu qui est à l'œuvre dans l'histoire du peuple d'Israël, dans la vie, la mort et la résurrection de Jésus et dans l'annonce du salut aux nations païennes. Il ne saurait donc y avoir de contradiction entre les différentes étapes de ce seul et unique projet.

Un exemple caractéristique de ce type de lecture est mis dans la bouche de Jésus lui-même en Luc 4,16-22. Après avoir lu un extrait du livre d'Isaïe, Jésus déclare: «Aujourd'hui s'accomplit à vos oreilles ce passage de l'Écriture (Luc 4,21). Dire qu'un texte de l'Écriture s'accomplit (littéralement est rempli), c'est sousentendre que jusque-là il n'avait pas trouvé sa complète réalisation et qu'il fallait attendre l'avènement du jour . $\mathrm{J}$ ", l'aujourd'bui pour voir se déployer toute la plénitude de sens qu'il contenait en germe.

Cette conviction fondamentale n'est pas formulée de manière théorique par les premiers auteurs chrétiens. Elle conditionne cependant la manière dont les Écritures juives, déjà reconnues 
comme normatives à l'époque de la naissance de l'Église, vont être utilisées dans l'élaboration du discours chrétien. Les textes de la Bible hébraïque et de la Bible grecque, de même que les événements auxquels ces textes se rapportent, sont repris, dans les écrits chrétiens, comme des annonces de ce qui se réaliserait avec la venue de Jésus ${ }^{8}$.

Une telle attitude n'évacue pas, en principe, le sens premier des textes et des événements, mais suppose qu'ils sont susceptibles de recevoir un supplément de sens. Dans la pratique cependant, la tendance se fit jour assez rapidement à mettre de côté le sens obvie des textes pour ne plus voir en eux que des annonces. Les textes hérités de la tradition juive deviennent ainsi peu à peu des rébus plus ou moins explicites, qu'il s'agit de décoder pour pouvoir les appliquer aux réalités nouvelles de la foi en Jésus.

L'utilisation chrétienne des textes bibliques consiste donc à abolir, d'une certaine manière, la distance qui sépare le texte du lecteur. Puisque le texte ne trouve sa pleine réalisation que dans l'aujourd'bui, c'est dans cet aujourd'bui que se fait la lecture. Le lecteur ne va pas à la rencontre du texte en tenant compte du fossé séparateur, mais il amène directement le texte à lui: l'aujourd'but original du texte est remplacé par l'aujourd'bui du lecteur. Du sens originel du texte on ne retient que ce qui est lu comme anticipation de la réalisation finale. Pour employer une comparaison, en partie inadéquate comme toute comparaison, on pourrait dire que le texte est à son accomplissement ce que la maquette de l'architecte est à l'édifice construit.

\section{Les traditions interprétatives juives}

Philon d'Alexandrie est un contemporain de Jésus. Juif fervent, il est également instruit des courants philosophiques de son temps, surtout du platonisme. Son œuvre entière consiste à établir un pont entre les deux cultures dont il est l'héritier. La présentation de la méthode philonienne exigerait la rédaction de tout un ouvrage tant sa pensée est complexe et se développe selon de multiples directions. Le terme allégorie caractérise le

8 Voir, par exemple, l'utilisation faite par Paul des écrits se rapportant à l'Exode en 1 Cor 10. 
$\overline{66}$

mieux l'exégèse de Philon. Pour lui, les personnages, les événements, les lieux, tout est susceptible de devenir image de la réalité spirituelle.

C'est principalement dans le christianisme que Philon a exercé de l'influence, en particulier à travers la célèbre École d'Alexandrie (Clément et surtout Origène et ses continuateurs). Dans le judaïsme, son influence demeura marginale. Quelques années après sa mort, le judaïsme subit les contrecoups de deux secousses majeures qui orientèrent pour longtemps sa manière de comprendre sa propre identité et, en conséquence, son rapport avec ses textes fondateurs. Ces deux événements sont, d'abord la prise de Jérusalem et la destruction du Temple par l'armée romaine en 70 et, vers la même époque, l'émergence du christianisme comme réalité autonome mais puisant aux mêmes sources que le judaïsme. Face à ces chocs, le judaïsme de tendance pharisienne réagit en élaborant un système interprétatif connu sous le nom général d'exégèse rabbinique.

On ne peut aborder ici tous les aspects de cette exégèse. Elle plonge ses racines loin dans le passé, probablement dans la communauté des exilés rentrés à Jérusalem au sixième siècle avant notre ère. La tradition rabbinique se réfère à une Loi orale, présumée venir de Dieu par l'intermédiaire de Moïse, et transmise d'une génération à l'autre par une chaîne ininterrompue de Docteurs de la Loi. Cette Loi orale complète, interprète et actualise la Loi écrite pour la rendre accessible au croyant. Partant de la Loi écrite - la Torah - l'exégèse rabbinique développe d'une part une tradition proprement législative - la halakha - et une tradition édifiante ou morale - Ia aggadah. Ces développements seront compilés d'abord dans la Mishna et dans les recueils complémentaires puis repris et commentés dans les deux Talmud qui à leur tour donneront lieu à toute une littérature interprétative.

Les règles qui ont présidé à l'élaboration de cette tradition ont varié du littéralisme le plus strict à la spéculation la plus fantaisiste; chaque école, chaque génération de docteurs ayant les siennes. Mais la compilation finale qui nous est parvenue n'a pas voulu choisir entre les différentes tendances; au contraire elle a conservé les interprétations provenant de toutes les écoles et illustrant toutes les méthodes. Il y a loin de l'exégèse de Philon d'Alexandrie à celle des compilateurs du Talmud. Pourtant, d'une certaine manière, on peut dire qu'une même préoccupation a animé tous ces lecteurs des Écritures: rendre au texte une 
actualité nouvelle en l'interprétant, en déduisant de la lettre des conséquences applicables aux situations nouvelles vécues par les lecteurs. Par contre il existe aussi, entre les deux courants, une différence fondamentale. Philon était un savant travaillant pour lui-même; la publication de ses œuvres n'avait d'autre autorité que celle de leur propre valeur. Il ne prétendait pas imposer son interprétation comme universellement valable et, nous l'avons déjà dit, il fut peu suivi à l'intérieur du judaïsme. Les rabbins à l'origine de la Mishna et des Talmud sont les chefs intellectuels et religieux de la communauté juive après la ruine du Temple. Leur préoccupation est d'éduquer le peuple à la pratique exacte de la Loi. Leurs interprétations deviennent normatives au même titre que la Loi elle-même à laquelle elles finissent parfois par se substituer. Ainsi l'interprétation de la Bible engendre un nouveau canon qui, sans prétendre éliminer le premier, en vient à occuper une place aussi importante, sinon plus, que le premier qu'il s'agissait au départ de rendre plus accessible et plus actuel.

\section{L'exégèse chrétienne: lire la Bible en Église}

Il ne semble pas que le principe de l'addition des écrits chrétiens à ceux qui ont été hérités de la tradition juive ait fait problème. Dès la fin du premier siècle de notre ère ou le début du deuxième, les lettres attribuées à Paul sont assimilées aux Écritures ${ }^{9}$. Tout au plus note-t-on un flottement concernant la liste des ouvrages à admettre. Une nouvelle collection d'Écrits notre Nouveau Testament - acquiert une autorité supérieure à celle de l'Ancien dont il est l'accomplissement, ainsi que nous l'avons déjà vu ${ }^{10}$. Pour les chrétiens, interpréter les Écritures renvoie donc à l'ensemble du corpus composé des deux Testaments qui doivent être éclairés sans cesse l'un par l'autre.

En simplifiant beaucoup, on peut dire que des origines à la Réforme, il n'a pas existé dans l'Église, de question biblique dans le sens où la Bible aurait fait l'objet d'une étude pour elle-même, sans référence à la théologie et à la vie ecclésiale. Bien sûr, cette

9 Voir 2 P 3,16.

10 Voir aussi l'article de Jean-Yves Thériault. En contexte juif, la Mishna, la Tosephta et les Talmud, malgré leur grande autorité, ne sont pas mis sur le même pied que les Ecritures elles-mêmes, au moins sur le plan théologique. 
68

longue période d'un millénaire et demi a connu de nombreux exégètes bibliques, et de très grands. Des méthodes d'étude ont été mises au point; des débats passionnés ont surgi entre partisans et adversaires de telle ou telle interprétation. Mais toute cette activité se déroule sur un horizon commun: la Bible appartient à l'Église; sa lecture et son interprétation se font en Église. Son message ne peut être que cohérent de la première page à la dernière et il est le même que celui qui est transmis par l'Église.

Même si les auteurs de l'Antiquité chrétienne et du Moyen Âge étaient conscients du fait que la Bible avait été écrite par des auteurs humains, vivant dans une culture différente de la leur et s'exprimant dans des langues autres, leur manière de lire abolissait, à toutes fins utiles, cette distance. L'iconographie, peut être plus encore que la littérature, nous renseigne sur cette proximité du texte biblique par rapport aux croyants aussi bien en Orient qu'en Occident. Les personnages bibliques des icônes byzantines portent les vêtements de la cour impériale; les vitraux et les enluminures du Moyen Âge latin montrent les personnages bibliques en costumes européens et dans des décors qui reproduisent les paysages de l'Europe médiévale, avec ses châteaux, ses villes et ses villages. Pour les chrétiens de ces époques, surtout pour les croyants ordinaires, les personnages bibliques sont des contemporains, des personnages familiers qui font partie de leur environnement naturel.

Ce qui est vrai dans l'expression populaire de la foi se retrouve également dans la recherche théologique. Le Moyen Âge latin développe un système d'interprétation basé sur les quatre sens de l'Écriture: sens littéral, sens allégorique, sens anagogique (ou eschatologique), sens tropologique (ou moral). Cette démarche se fonde sur la conviction que Dieu parle aux croyants à travers les Écritures, donc que celles-ci ont du sens dans l'aujourd'bui des lecteurs. Le texte devient en quelque sorte contemporain de celui qui le lit. Même la recherche du sens littéral se fait à travers le prisme de la foi chrétienne et, en particulier, de son expression théologique scolastique. Il ne s'agit donc pas, à proprement parler, d'une recherche de type historique visant à remonter aux origines du texte, mais d'une actualisation du texte en son sens le plus obvie, d'une transposition dans l'aujourd'bui du lecteur et de l'Église. 


\section{Lecture de la Bible et nouvelle vision du monde}

Ce qui change le plus radicalement à partir de la Renaissance c'est l'image que l'être humain se fait de lui-même, du monde dans lequel il vit et de son rapport avec ce monde.

Durant toute l'Antiquité et le Moyen Âge, Dieu se profile à l'horizon du monde comme sa cause première et sa fin ultime. Son intervention dans l'histoire, quelles qu'en soient les modalités, n'est pas remise en question. Depuis la Renaissance, se développe une compréhension de l'univers selon laquelle les événements du monde, comme les phénomènes naturels, se déroulent selon des lois qu'il est possible aux humains de découvrir. Le principe de causalité, jusque-là situé en dehors du monde, est désormais perçu comme intra-mondain.

On devine facilement les bouleversements qu'un tel changement entraîne dans la lecture d'une littérature qui se définit elle-même comme Parole de Dieu. Si la cause de toutes choses se situe à l'intérieur du monde lui-même, la collection biblique doit être étudiée comme n'importe quelle œuvre littéraire. Les règles mises au point pour l'étude des autres ouvres anciennes peuvent et doivent être appliquées à la Bible et, par ailleurs, la relation du texte biblique au peuple croyant doit être repensée en termes nouveaux: si la Bible est un discours humain, conditionné par les circonstances particulières de l'époque de sa formation, comment peut-il être normatif pour la foi ? comment peut-il être encore Parole de Dieu dans l'aujourd'hui des lecteurs.

\section{Le conflit de la foi et de la raison}

La problématique que nous venons d'évoquer se situe à l'intérieur d'un conflit plus vaste. De l'affaire Galilée au cas du père Teilhard de Chardin, pour ne citer que les péripéties les plus connues, ce sont trois siècles de tâtonnements et souvent d'affrontements entre, d'une part, les tenants de la primauté de l'autorité divine fondée sur une révélation donnée une fois pour toutes et, d'autre part, les partisans d'un examen systématique de tout le connaissable à la lumière de la raison. Dans le domaine biblique, ce débat a opposé les partisans d'une étude scientifique de la Bible selon les critères appliqués aux autres œuvres littéraires de la même époque et les défenseurs de l'autorité divine des $\hat{E} c r i-$ tures conçues comme une Parole de Dieu immuable. Dans 
70

l'Église catholique le conflit a culminé, au début du vingtième siecle, dans la crise Moderniste. Mais les Églises protestantes ont connu elles aussi des déchirements à mesure que les autorités religieuses voyaient l'interprétation de la Bible leur échapper pour devenir l'apanage des spécialistes. Le conflit, encore actuel dans certaines régions des États-unis, entre partisans de l'évolution des espèces et défenseurs de la Création en sept jours se situe dans le prolongement de la même problématique.

Pour la première fois de l'histoire, la lecture de la Bible cesse de se faire à l'intérieur d'une Église, comme une discipline théologique. Elle devient une démarche autonome, ayant en ellemême sa finalité. Non pas que les exégètes de cette époque aient voulu dès le point de départ rompre avec leur Église, mais ils ont entrepris leur recherche en essayant de mettre de côté toutes les connaissances, théologiques ou autres, véhiculées par la tradition croyante, afin de revenir au texte seul, dans sa pureté originelle.

Cette démarche se situe évidemment dans la foulée des Lumières et du courant positiviste. Elle pose en principe la possibilité de connaître objectivement le réel, à la condition de posséder la méthode appropriée. Lorsque l'objet à connaître est un texte, il faut mettre au point une approche permettant à tout lecteur, peu importent ses convictions ou ses interrogations personnelles, de découvrir le sens voulu par l'auteur. Établi par l'application des méthodes appropriées, ce sens doit pouvoir s'imposer de manière universelle puisqu'il est présumé correspondre à l'intention de l'auteur. Cette démarche, au moins dans son principe sinon toujours dans son application, se veut exclusive. Une fois le sens établi d'une manière aussi objective que possible, toutes les autres interprétations paraissent des fantaisies tirées de l'imagination des exégètes ou de leurs préjugés dogmatiques.

\section{Une révolution}

Le passage des approches traditionnelles à cette nouvelle attitude envers le texte a constitué une sorte de révolution copernicienne. Pour la première fois peut-être, le lecteur ne veut plus amener le texte à lui, le transposer dans son propre aujourd'bui, mais il cherche à se transporter dans l'aujourd'bui primitif du texte pour être sur le même pied que le premier lecteur à qui le texte s'adressait directemènt. La distance est franchie par le lecteur qui se transporte vers le texte! 
L'ensemble des approches et des moyens d'analyse mis en œuvre par cette attitude nouvelle est connu sous le nom de méthode bistorico-critique. Malgré la résistance rencontrée dans certains milieux à cause des remises en questions qu'elle impliquait, elle a réussi à s'imposer comme la manière de faire de l'exégèse biblique. Comme telle, elle a régné en maîtresse dans les facultés de théologie en Occident durant la plus grande partie du vingtième siècle.

La méthode historico-critique ${ }^{11}$ a rendu à la lecture de la Bible d'inestimables services. Elle a d'abord redonné la priorité au texte lui-même. La démarche historique a pour but de comprendre ce que dit le texte, et non pas les vérités théologiques, spirituelles ou morales qu'on pourrait en déduire par des procédés plus ou moins rigoureux. De plus, on prend conscience que les auteurs de ces textes s'expriment selon des conventions littéraires propres à leur temps et à leur culture, qui peuvent être différentes des nôtres. D'où la nécessité de connaître le milieu qui a vu naître l'écrit et aussi son genre littéraire. Ces connaissances permettent de relativiser ce qui est affirmé dans le texte, de faire la distinction entre le message transmis et l'expression littéraire à travers laquelle il est véhiculé.

En rendant la priorité au texte lui-même, la méthode historico-critique empêche le lecteur de faire du texte un prétexte pour développer des idées qu'il tire d'une autre source. En objectivant le texte et en définissant clairement les voies d'approches, elle rend possible une forme de contrôle des résultats obtenus. Par contre, en mettant l'accent uniquement sur le sens premier, historique, du texte, elle laisse sans réponse la question de son actualité. Le rapport entre le texte ancien, historiquement situé dans son contexte, et l'aujourd'bui du lecteur risque de devenir de plus en plus difficile à établir. D'où la question posée par beaucoup de croyants: "Ces vieux textes ont-ils encore du sens pour nous aujourd'hui? peuvent-ils être encore Parole de Dieu?. Et même en dehors de toute perspective croyante, la question peut aussi être posée: "Le sens premier historique d'un

11 Bien qu'il s'agisse en fait d'un ensemble de méthodes plus ou moins convergentes, nous employons l'expression au singulier car toutes ces approches ont en commun leur attitude envers le texte. Pour ne pas entrer dans des développements qui déborderaient notre cadre, nous ne distinguons pas les différentes écoles historico-critiques. 
texte épuise-t-il toute la signification? est-il possible de lire aujourd'hui ce texte d'une manière différente de celle du premier lecteur, sans pour cela trahir le texte et son auteur?"

\section{Les remises en question actuelles}

À vrai dire, l'objectivation du texte par la méthode historicocritique n'a jamais été aussi absolue que peuvent le laisser croire les lignes qui précèdent. Un exégète comme Rudolph Bultmann écrivait en 1957:

[...] il n'y a pas d'exégèse sans présupposition, l'exégète n'étant pas une table rase mais abordant au contraire le texte avec ses questions propres, ou plutôt avec sa façon propre de poser le problème, et ayant une certaine notion de la chose dont il s'agit dans le texte. ${ }^{12}$

Cette déclaration de Bultmann ne se veut pas une remise en question des méthodes de la tradition historico-critique. Elle amorce tout de même un virage dans l'attitude des exégètes de métier: le centre d'intérêt se déplace peu à peu de l'auteur du texte - et de ce qu'on croit être ses intentions - vers le lecteur. Sans renoncer aux méthodes d'analyse déjà éprouvées, on cherche davantage à comprendre pour qui les textes ont été écrits, on se demande à quels besoins ils pouvaient répondre, quelles crises au sein de leur communauté d'origine ont été l'occasion de leur rédaction?

Du public lecteur original on peut passer au public lecteur d'aujourd'hui et poser la question: comment le lecteur moderne trouve-t-il du sens dans ces textes anciens? Il ne s'agit pas d'un pur retour en arrière, ni d'une mise de côté de tout l'héritage des études bibliques depuis des générations. Cette question prend acte du fait que la distance existe entre le texte et le lecteur mais - et c'est la différence fondamentale avec la méthode historico-critique - elle essaie de construire un pont au-dessus de ce fossé, non pour essayer de le franchir dans un sens ou dans l'autre mais pour relier les deux rives en respectant chacune pour ce qu'elle est dans son originalité par rapport à l'autre.

12 R. Bultmann, :Une exégèse sans présupposition est-elle possible? , ,Foi et comprébension. Eschatologie et démythologisation, traduit par André Malet, Paris, Seuil, 1969, p. 167. 
Depuis une génération environ, on se penche sur ce problème de l'implication du lecteur dans la détermination du sens d'un texte, en particulier d'un texte biblique.

Le texte biblique peut être vu comme un appel a la capacité d'imagination du lecteur et à sa recherche d'un sens cohérent avec sa propre situation dans le monde. ${ }^{13}$

Cette citation illustre bien la tendance actuelle: le sens d'un texte n'est pas seulement donné, il est construit, c'est-à-dire qu'il s'établit dans le rapport dynamique et sans cesse renouvelé entre le lecteur et le texte. Il ne s'agit pas vraiment d'une découverte récente, puisque toutes les générations de lecteurs de la Bible ont, chacune à sa manière, tenu compte de ce fait. Le développement de méthodes de lecture inspirées par la philosophie du langage a cependant permis de mettre en lumière ce phénomène et d'en donner une interprétation plus articulée.

\section{Vers l'avenir}

Dégageons une constante de ce tour d'horizon historique forcément incomplet: aucune approche du texte biblique ne peut prétendre en épuiser tout le sens. Chaque époque a mis au point des méthodes qui lui permettaient d'actualiser le texte dans un contexte donné. Chaque génération de lecteurs et de relecteurs a dû trouver des moyens pour franchir la distance la séparant du texte. Il n'y a pas raison de croire que ce mouvement devrait maintenant s'arrêter. Les méthodes du passé n'ont sans doute pas donné tous les résultats dont elles sont capables et des méthodes nouvelles seront sans doute mises au point pour répondre à des besoins nouveaux et encore inédits.

Qu'on aborde le texte biblique comme Parole de Dieu, comme monument littéraire ou comme témoignage historique, son interprétation est toujours un événement qui modifie non seulement le rapport du lecteur au texte mais aussi le rapport du lecteur au monde. En ce sens, la lecture de la Bible est une aventure jamais terminée, c'est donc une histoire à suivre...

13 Edgar V. McKnight, Post Modern Use of the Bible. The Emergence of Reader Oriented Criticism, Nashville, Abingdon Press, 1988, p. 107. 